\title{
Orthogonal modular biosynthesis of nanoscale conjugate vaccines for vaccination against infection
}

\author{
Xin Li, Chao Pan $(\varangle)$, Peng Sun, Zhehui Peng, Erling Feng, Jun Wu, Hengliang Wang ( $\varangle)$, and Li Zhu \\ State Key Laboratory of Pathogen and Biosecurity, Beijing Institute of Biotechnology, Beijing 100071, China \\ (c) Tsinghua University Press and Springer-Verlag GmbH Germany, part of Springer Nature 2021 \\ Received: 16 April 2021 / Revised: 17 June 2021 / Accepted: 24 June 2021
}

\begin{abstract}
Conjugate vaccines represent one of the most effective means for controlling the occurrence of bacterial diseases. Although nanotechnology has been greatly applied in the field of vaccines, it is seldom used for conjugate vaccine research because it is very difficult to connect polysaccharides and nanocarriers. In this work, an orthogonal and modular biosynthesis method was used to produce nanoconjugate vaccines using the SpyTag/SpyCatcher system. When SpyTag/SpyCatcher system is combined with protein glycosylation technology, bacterial O-polysaccharide obtained from Shigela flexneri $2 \mathrm{a}$ can be conjugated onto the surfaces of different virus-like particles (VLPS) in a biocompatible and controlled manner. After confirming the excellent lymph node targeting and humoral immune activation abilities, these nanoconjugate vaccines further induced efficient prophylactic effects against infection in a mouse model. These results demonstrated that natural polysaccharide antigens can be easily connected to VLPS to prepare highly efficient nanoconjugate vaccines. To the best of the researchers' knowledge, this is the first time VLP-based nanoconjugate vaccines are produced efficiently, and this strategy could be applied to develop various pathogenic nanoconjugate vaccines.
\end{abstract}

\section{KEYWORDS}

Shigela flexneri 2a, O-polysaccharide, virus-like particle, SpyTag/SpyCatcher system, nanoconjugate vaccines

\section{Introduction}

Vaccines play an important role in the prevention and control of infectious diseases [1]. With the development of genetic engineering technology, many vaccines now consist of protein subunits rather than whole cells. Although the safety of subunit vaccines has been demonstrated, their immunogenicity is greatly reduced compared to whole cell vaccines. Also the duration of their immunity is short [2-5]. Therefore, adjuvants and appropriate delivery systems are needed to enhance immune responses to subunit vaccines [6-11]. Inspired by the structure and function of pathogens, biomimetic particles were developed for vaccine applications to simultaneously deliver antigens and stimulate immune. This can improve the stability of vaccine antigens, facilitate targeting to draining lymph nodes (dLNs) [12-14], and enhance cross-presentation to increase immunogenicity $[15,16]$.

Biological nanoparticles have dominated trials of nanovaccines. Inorganic and chemically synthesized nanoparticles including metals $[17,18]$ and synthetic polymers [19-21] are being explored in preclinical research. Virus-like particles (VLPs) are self-assembling nanoparticles that mimic the structural organization of viruses. VLPs have a highly repetitive surface structure that promotes $B$ cell receptor aggregation and complements fixation, thereby activating humoral and cellular immune responses [22]. VLPs can be used to display antigens through genetic fusion [23-25] or chemical conjugation $[26,27]$. In recent years, the split-protein (SpyTag/SpyCatcher) conjugation system has attracted significant attention. This system exploits the isopeptide bond formed spontaneously between Lys and Asp residues in the two split units of Streptococcus pyogenes fibronectin-binding protein CnaB2. The split units (SpyCatcher protein and SpyTag peptide) can form highly stable amide bonds within $1 \mathrm{~h}$ in a solution $[28,29]$. This system provides new opportunities for the modular synthesis of nanovaccines and has been used to display a variety of protein antigens on the surface of bacteriophage AP205 [30-32]. Spy-AP205 has been studied as a scaffold for various candidate vaccines against multiple targets, including malaria and cancer [33].

Bacterial surface polysaccharides often possess desirable characteristics as candidate antigens including surface exposure, immunogenicity, and mechanistic role in the pathogenesis of disease [34]. Therefore, polysaccharide antigens are attractive for the development of bacterial vaccines. The traditional method of preparing conjugate vaccines is through chemical conjugation $[35,36]$, in which polysaccharides are purified separately from the pathogenic organism and the carrier protein from another organism. The polysaccharides are activated at random positions or at their reducing ends and mixed with the carrier protein to cross-link them [37]. In recent years, there have been enormous advances in the development of conjugate vaccines for prophylactic applications. The development of nanoscale carrier proteins has dramatically improved the immune responses elicited by conjugate vaccines. In one emerging method, chemically synthesized oligosaccharides are attached to the pre-assembled Q $\beta$ surface via copper-catalyzed azide-alkyne cycloaddition reactions [18]. The limitation of 
this method is the difficulty of synthesizing complex oligosaccharides. On the contrary, protein-glycan coupling technologies (PGCT) can be used to produce polysaccharide antigens with complex structures and couple them with carrier proteins in bacterial cells in vivo. In our previous work, we successfully transferred polysaccharides to self-assembled nanoparticles using glycosyltransferases expressed in the bacterial periplasm to generate nanoconjugate vaccines in a single step. This reduced the costs and time of preparation $[38,39]$. However, the assembly of nanoparticles in the periplasm affects the total yields of nanoconjugate vaccines. Furthermore, this strategy requires different purification processes and quality control standards for producing nanovaccines against different pathogens.

Herein, we established an orthogonal and modular nanoconjugate vaccine preparation method combining the advantages of the above two methods. Polysaccharide antigens and virus-like particles are expressed in attenuated pathogenic bacteria (Shigela flexneri) and virulent Escherichia coli cells, respectively. The polysaccharide antigens are then coupled to the surface of VLPs (bacteriophage AP205 or Q $\beta$ ) using the SpyTag/SpyCatcher system. This method enables the uncoupled expression of polysaccharide antigens and proteinaceous nano-scaffolds, making the scaffold particles validated and stored in advance, and also react with diverse antigens as needed. To evaluate the resulting nanovaccines, immunogenicity was assessed by immunizing $\mathrm{BALB} / \mathrm{c}$ mice and assessing serum reactivity against polysaccharide antigens. The advantages of nanoconjugate vaccines include enhanced dendritic cell (DC) maturation, effective delivery to lymph nodes, and activation of germinal center (GC) B cells. We further demonstrated that the nanoconjugate vaccines induced high-titer antibodies against bacterial lipopolysaccharide (LPS) and had strong prophylactic effects against infection in a mouse model. Our method provides an attractive platform technology combining SpyTag/SpyCatcher system and PGCT to quickly generate nanoconjugate vaccines.

\section{Results and discussion}

\subsection{Nanoconjugate vaccine preparation and charac- terization}

Bacterial surface $O$ antigen polysaccharides (OPS) can elicit protective antibodies. In previous studies, a Shigella conjugate vaccine prepared with cholera toxin B (CTB) as the carrier protein elicited protective antibodies in mice [40]. To achieve orthogonal and modular production of polysaccharides and proteins, we used PGCT to prepare SpyCatcher-OPS (SC-OPS) and then covalently attached the resulting polysaccharides to the surface of VLPs using the SpyTag/SpyCatcher system. To generate SpyTag-VLPs (ST-VLPs), we genetically fused SpyTag to the $\mathrm{N}$-terminus of the RNA bacteriophages AP205 or Q $\beta$ and added a C-terminal His 6 tag for downstream applications (Fig. 1(a)). ST-VLPs were expressed in E. coli BL21 (DE3) cells (Fig. 1(b)). ST-VLPs were purified from cell lysates by affinity chromatography followed by dialysis using a $100 \mathrm{kDa}$ molecular weight cut-off (MWCO) membrane. Dynamic light scattering (DLS) of the purified extracts revealed the formation of ST-AP205 and ST-Q $\beta$ conjugates approximately $30 \mathrm{~nm}$ in diameter. A single band of the expected size $(\sim 17 \mathrm{kDa})$ was observed on a sodium dodecylsulfate (SDS)-polyacrylamide

(a)

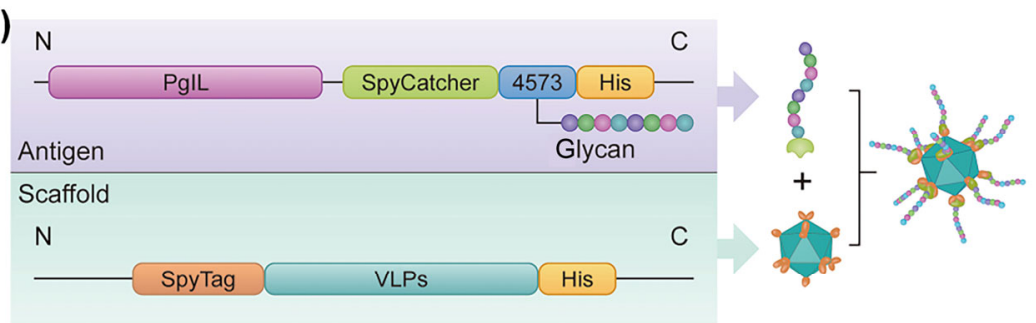

(b)

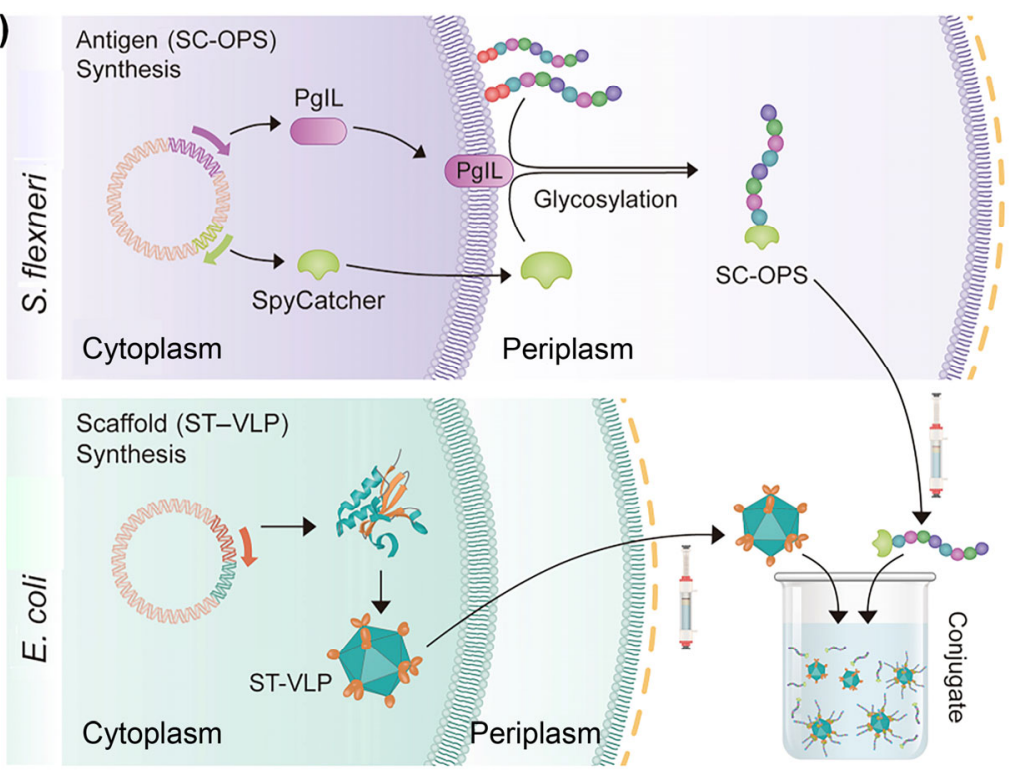

Figure 1 Design principles of the nanoscale conjugate vaccine platform. (a) Modular design scheme of construct design particle and glycoprotein. (b) Schematic illustration of nanovaccine preparation. S. flexneri was transformed with the pET-PglL-SpyCatcher ${ }^{473 \mathrm{C}}$ plasmid to prepare the glycoprotein (SC-OPS). SpyTag was genetically fused to the N-terminus of VLP proteins (ST-VLP) and expressed in E. coli. After mixing, ST-VLPs formed spontaneous isopeptide bonds with SC-antigens (OPS). 
gel electrophoresis (PAGE) gel stained with Coomassie blue

(Fig. S1 in the Electronic Supplementary Material (ESM)).

Next, we used PGCT to prepare SC-OPS. First, S. flexneri strain 301DWP cells (an attenuated strain of S. flexneri 2a 301) were transformed with the expression plasmid pET-PglLSpyCatcher ${ }^{4573 C}$. The attenuated 301DWP strain lacks the virulence plasmid and $\mathrm{O}$ antigen ligase [40]. After induction with isopropyl $\beta$-D-1-thiogalactopyranoside (IPTG), the OPS was transferred to glycosylation sequence 4573 via catalysis with PgIL to yield glycoprotein SC-OPS (Fig. 1(b)). The glycoprotein was purified by affinity chromatography and size exclusion chromatography. Coomassie blue staining of SDS-PAGE gels revealed a typical extended ladder, with each band corresponding to a different number of polysaccharide repeat units (pentasaccharides) (Fig. S2 in the ESM). To improve the homogeneity of the antigen, a glycoprotein with 11-17 repeated pentaoligomeric sugar units was selected for subsequent binding experiments. Notably, we did not apply any additional interventions during polysaccharide synthesis, which could ensure the natural configuration of the polysaccharide antigen.

To test if the recombinant ST-VLPs could form a spontaneous isopeptide bond with antigens through their SpyCatcher moieties, individual ST-VLPs were mixed with SC-OPS and formation of VLP-antigen conjugates was confirmed by SDS-PAGE. After mixing with ST-VLPs, a clear shift in the molecular weight of the SC-OPS band was observed on SDS-PAGE, indicating covalent bond formation (Fig. 2(a)). In subsequent experiments, unconjugated components were removed by size exclusion chromatography (Fig. S2 in the ESM). After confirming the formation of covalent ST-VLP + SC-OPS conjugates, we used immunoblotting for further analysis. Both the ST-AP205 + SC-OPS (AP205-OPS) and ST-Q $\beta$ + SC-OPS (Q $\beta$-OPS) conjugates strongly reacted with anti-His $_{6}$ and anti-OPS monoclonal antibodies (Fig. 2(b)). Furthermore, transmission electron microscopy images of the AP205-OPS and Q $\beta$-OPS conjugates showed well-dispersed, spherical nanoparticles (Fig. 2(c)). Quantitation of the purified AP205-OPS and Q $\beta$-OPS conjugates by DLS showed uniform peaks of hydrodynamic radius $46.3 \pm 1.4 \mathrm{~nm}$ and $43.2 \pm 1.1 \mathrm{~nm}$, respectively (mean \pm standard deviation (SD), $n=3$ ) (Fig. 2(d)). As expected, this size was larger than the hydrodynamic radius of the ST-VLPs because of the presence of polysaccharide chains on the VLP surface.

The inactivation of vaccines because of cold chain failure is a major challenge in the developing world [41]. Therefore, vaccines that can be stored at room temperature would be highly desirable. The thermal stability of the nanoconjugate vaccines was evaluated using a thermal shift assay. The samples were depolymerized gradually as the temperature increased from 25 to $99^{\circ} \mathrm{C}$, as the dye bound to hydrophobic amino acid residues, it emitted a fluorescence signal. As shown in Fig. 2(e), AP205-OPS and Q $\beta$-OPS depolymerized around 69 and $65{ }^{\circ} \mathrm{C}$, respectively. The particle integrity of both nanoconjugate vaccines did not change following storage at $30^{\circ} \mathrm{C}$ for 20 days (Fig. S3 in the ESM). The stability of the nanoconjugate vaccines to lyophilization was further evaluated. Both nanoconjugate vaccines could be lyophilized and reconstituted without degrading as shown by SDS-PAGE (Fig. S4 in the ESM). These results confirmed the high stability of the nanoconjugate vaccines.

\subsection{DC maturation in vitro promoted by nanovaccines}

Antigen uptake and activation of antigen-presenting cells (APCs, including macrophages and DCs) are key steps in the induction of effective immune responses [42]. VLPs can promote the recruitment of DCs to dLNs and promote DC maturation [14]. To assess whether the nanoconjugate vaccines could enhance bone marrow-derived DC (BMDCs) maturation, BMDCs were incubated with phosphate-buffered saline (PBS), OPS, SC-OPS, AP205-OPS, or Q $\beta$-OPS. Expression of BMDCs maturation markers, including costimulatory molecules and cytokines, was assessed by flow cytometry and enzyme-linked immunosorbent assay (ELISA), respectively. Compared with

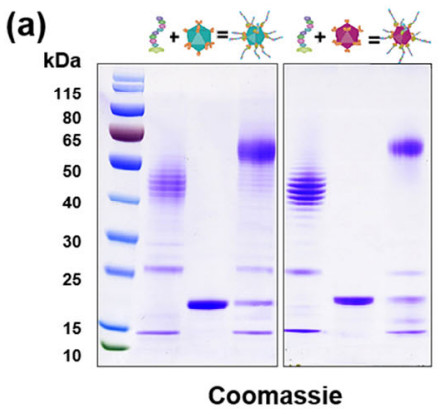

(d)
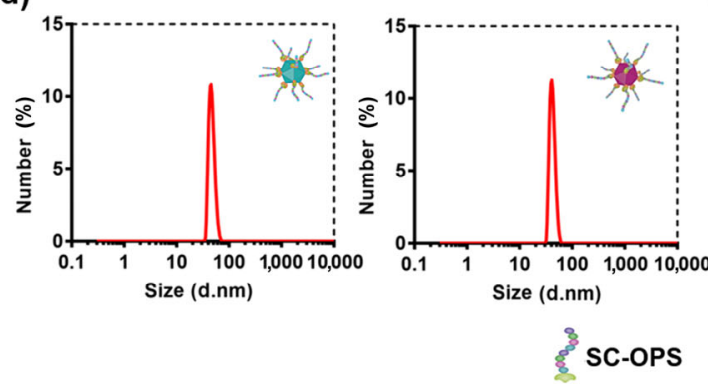

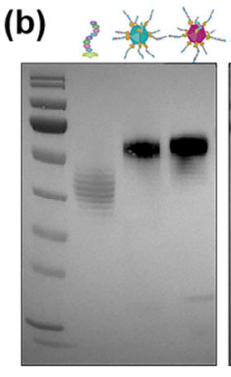

Anti-his

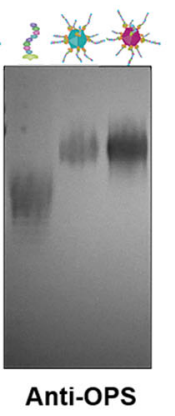

(c)

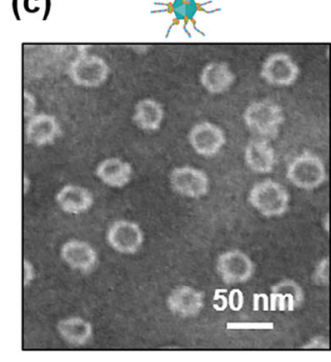

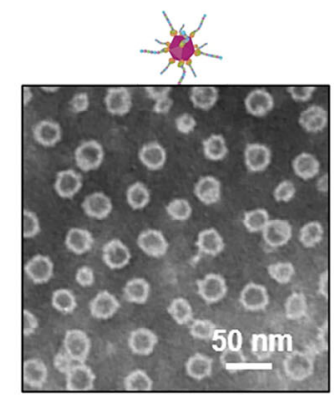

(e)
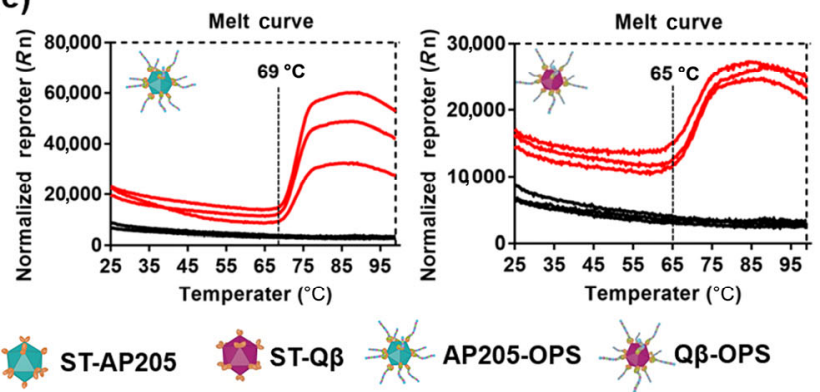

Figure 2 Preparation and characterization of nanoconjugate vaccines. (a) SDS-PAGE analysis of covalent complex formation between SC-OPS and SpyTag-AP205/Q $\beta$ at $4{ }^{\circ} \mathrm{C}$ overnight. (b) SC-OPS and nanovaccines were detected by western blotting using antibodies against His 6 and $S$. flexneri 2 a OPS. (c) Transmission electron microscopy images of AP205-OPS and Q $\beta$-OPS. Scale bar: $50 \mathrm{~nm}$. (d) The hydrodynamic radii of the AP205-OPS and Q $\beta-O P S$ nanoconjugate vaccines were determined by dynamic light scattering. (e) Thermal stability of the AP205-OPS and Q $\beta$-OPS nanoconjugate vaccines was assessed using a thermal shift assay. 
PBS, OPS, or SC-OPS, AP205-OPS and Q $\beta$-OPS induced significant increases in the numbers of cells expressing MHC-II and the co-stimulatory molecules CD40, CD80, and CD86 (Fig. 3(a)). Moreover, AP205-OPS and Q $\beta$-OPS significantly increased the expression of interleukin (IL)-6, tumor necrosis factor (TNF)- $\alpha$, and IL-1 $\beta$, respectively (Fig. 3(b)). Mice were immunized and lymph nodes were obtained $24 \mathrm{~h}$ later to evaluate the effect of nanoconjugate vaccines on DCs maturation by flow cytometry. AP205-OPS and Q $\beta$-OPS significantly increased the expression of MHC-II, CD40, CD80, and CD86 on lymph node DCs (Fig. S5 in the ESM). These results clearly confirmed that the complex structures of polysaccharides do not affect the immune characteristics of VLPs.

\subsection{Lymph node drainage and GC formation enhanced by nanovaccines}

To investigate the retention and distribution of nanovaccines, firstly local reactions at injection sites were assessed. Sulfocyanine7 succinimidyl ester (Cy7-SE) fluorescent dye was used to covalently label AP205-OPS, Q $\beta$-OPS, and SC-OPS. $\mathrm{BALB} / \mathrm{c}$ mice were injected at the base of their tail and subjected to in vivo fluorescence imaging. As shown in Fig. 4(a), fluorescence decayed quickly at the injection sites $48 \mathrm{~h}$ after SC-OPS group was injected. In contrast, the sites of nanovaccines injection showed prolonged signals even after $72 \mathrm{~h}$. As the signal intensity of the antigens at the injection site decreased, they accumulated in dLNs. The SC-OPS signal was low in dLNs and degraded quickly. In contrast, AP205-OPS and Q $\beta$-OPS showed enhanced retention in dLNs, especially AP205-OPS (Fig. 4(b)). Thus, the nanovaccines were transported to local dLNs efficiently because of their particle size. The superior lymph node targeting of nanovaccines may have contributed to their immunogenicity.

The formation of GCs is essential for strong antibody responses and the affinity selection of GC B cells is dependent on $\mathrm{CD}^{+}{ }^{+} \mathrm{T}$-follicular helper (Tfh) cells $[43,44]$. AP205-OPS and $\mathrm{Q} \beta$-OPS immunization resulted in the enlargement of dLNs because of increased $\mathrm{T}$ cell proliferation (Fig. 4(c)). We further analyzed the proportions of $\mathrm{CD}^{+} \mathrm{T}$ cells in dLNs on the third-day post-injection and observed a significant increase in mice immunized with AP205-OPS and Q $\beta$-OPS but not with SC-OPS (Fig. 4(d)). These experiments also revealed that dLN Tfh cells in AP205-OPS- and Q $\beta$-OPS-immunized mice expanded on day 7 post-injection (Fig. 4(e)). Importantly, we found that the percentages of GC B cells within dLNs were significantly higher in nanovaccine-immunized mice compared with SC-OPS-immunized mice (Fig. 4(f)). These data showed that compared with OPS and SC-OPS, nanoconjugate vaccines more effectively promoted the localization and long-term persistence of antigens in dLNs, as well as the activation and maintenance of GCs. To visually confirm the expansion of GC $\mathrm{B}$ cells in response to nanovaccines, histological analysis was performed and it revealed high levels of the proliferative marker Ki-67 in the GC areas of dLNs from AP205-OPS- and $\mathrm{Q} \beta$-OPS-immunized mice (Fig. 4(g)). Therefore, nanoconjugate vaccines represent efficient antigen delivery platforms to enhance Tfh and GC B cell responses. These data suggested that the AP205-OPS and Q $\beta$-OPS were likely to stimulate potent humoral immune responses.

\subsection{Potent humoral immune responses elicited by nanovaccines}

After confirming the highly efficient immunostimulatory effects of the nanoconjugate vaccines, their safety was evaluated. $\mathrm{BALB} / \mathrm{c}$ mice were immunized subcutaneously with AP205-OPS and $\mathrm{Q} \beta$-OPS ( $25 \mu \mathrm{g}$ of polysaccharide equivalent) and continuously monitored for 30 days. The safety of the nanovaccines was assessed by measuring body weight, body temperature, inflammatory cytokines, and biochemical indicators (Fig. S6 in the ESM) of the mice. Having established the stability and safety of the nanovaccines, their ability to elicit immune responses was further explored. Six-week-old BALB/c mice were immunized with PBS, SC-OPS, SC-OPS + $\mathrm{Al}$ (aluminum

(a)
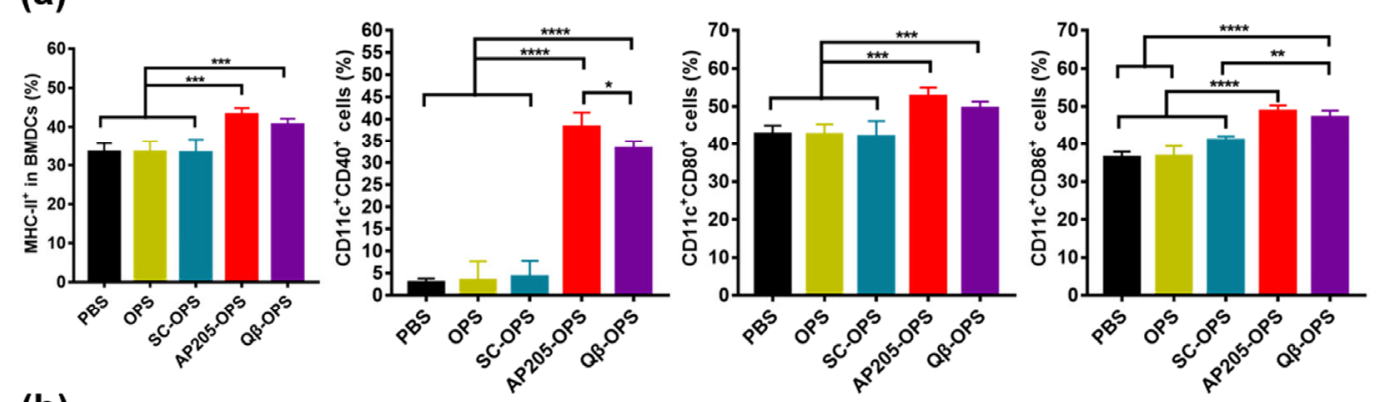

(b)
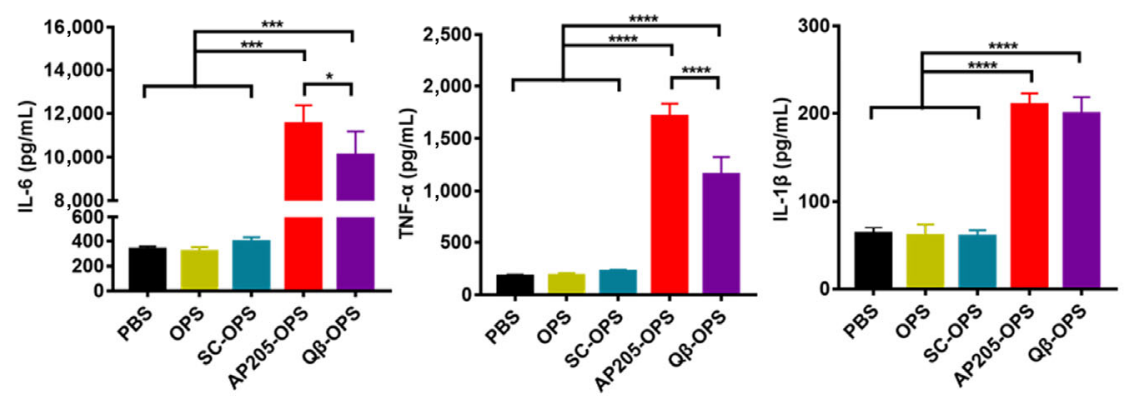

Figure 3 Nanovaccines induced BMDCs activation and maturation. BMDCs were incubated with AP205-OPS, Q $\beta$-OPS, SC-OPS, or OPS for 24 h, labeled with antibodies against CD11c, CD40, CD80, CD86, or MHC-II, and then analyzed by flow cytometry. (a) Percentages of MHC-II, CD40, CD80, and CD86 positive cells were calculated. (b) Secretion of IL-6, tumor necrosis factor- $\alpha$, and IL-1 $\beta$ in the supernatants of cultured BMDCs was measured by ELISA. Data were expressed as means \pm standard errors of the means $(n=4)$. Statistical analysis was performed using one-way analysis of variance with Dunn's multiple comparison test $\left({ }^{*} p<0.05,{ }^{* * *} p<0.001\right.$, and $\left.{ }^{\star * * *} p<0.0001\right)$. 

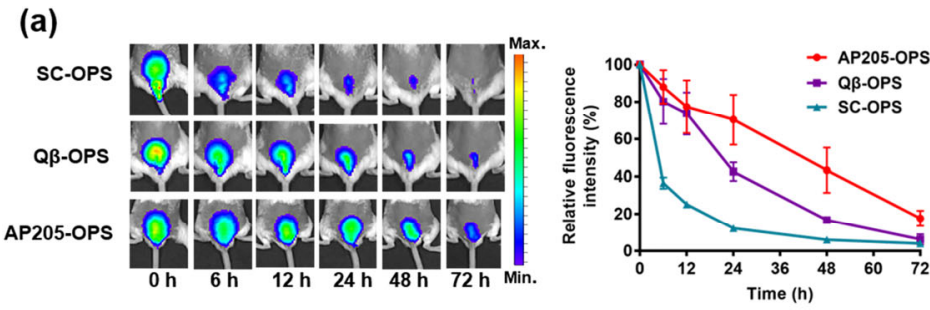

(b)
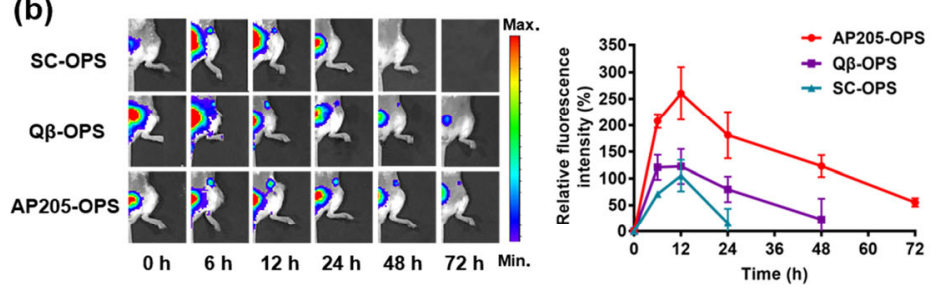

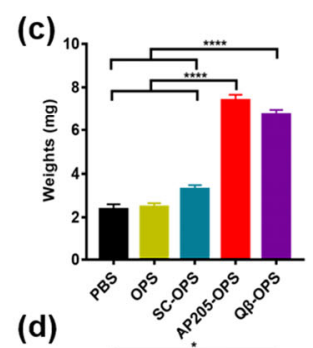

(d)

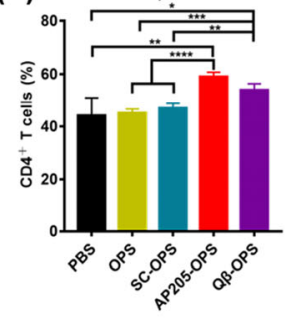

(e)
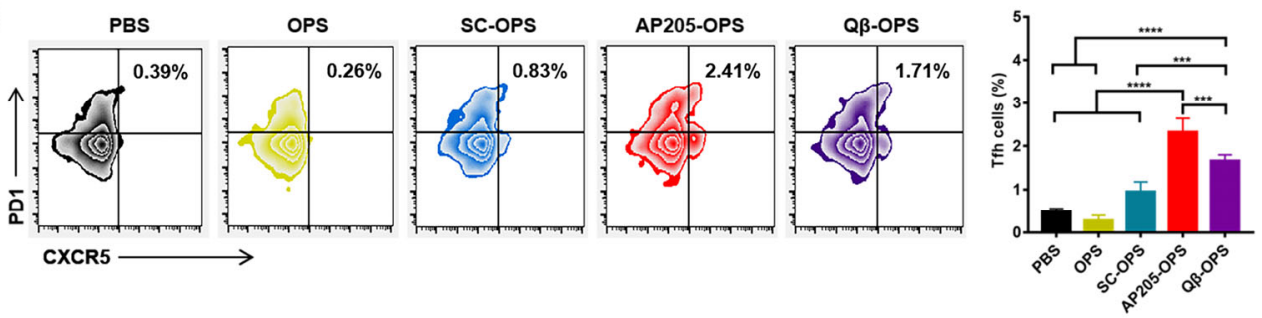

(f)
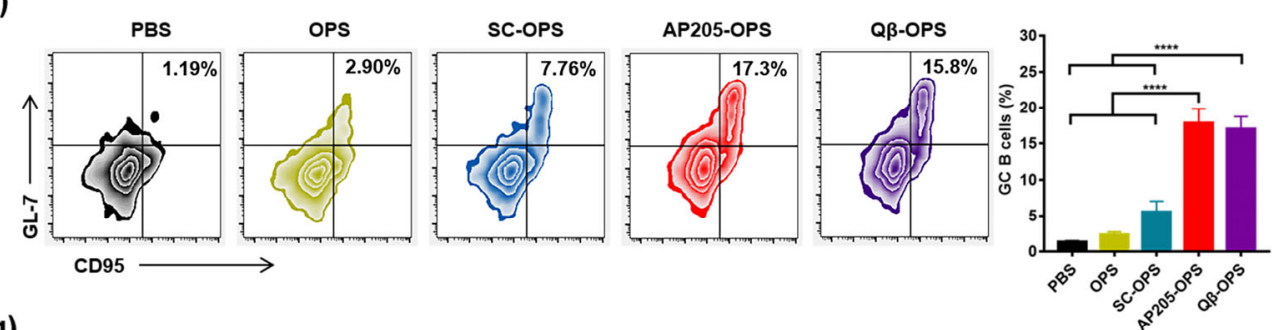

(g)
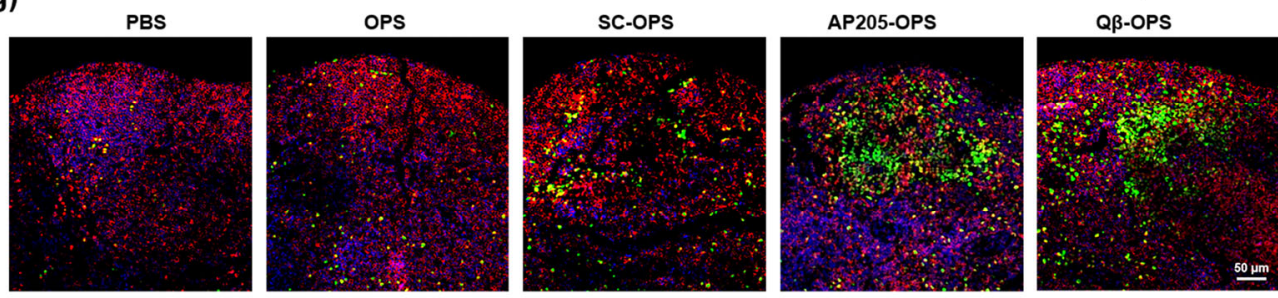

DAPI B220 Ki67

Figure 4 Nanovaccines show enhanced uptake in dLNs and induce efficient Tfh and GC responses. (a) and (b) BALB/c mice were subcutaneously injected with Cy7-labeled AP205-OPS, Q $\beta$-OPS, or SC-OPS. Antigen fluorescence was evaluated and quantitative signal intensity is shown for (a) injection sites and (b) dLNs. Data were presented as means \pm SDs $(n=3)$. (c) Weights of dLNs 3 days following immunization with different vaccines $(n=4)$. (d) Percentages of $\mathrm{CD} 4^{+} \mathrm{T}$ cells in dLNs 3 days post-vaccination $(n=4)$. (e) and (f) Proportion of Tfh cells $\left(\mathrm{CXCR} 5^{+} \mathrm{PD}-1^{+}\right.$cells among the CD4 ${ }^{+}$cell population) and GC B cells $\left(\mathrm{GL} 7^{+} \mathrm{CD} 95^{+}\right.$cells among B220 cells) in the dLNs as determined by flow cytometry 7 days post-vaccination $(n=4)$. The percentages and numbers of (e) Tfh and GC (f) B cells are shown. (g) Cryosections of dLNs were stained with anti-Ki67 antibody, anti-B220 antibody, and DAPI. Individual follicles were imaged by confocal microscopy (DAPI blue; B220 red; Ki67 green). Data were presented as means \pm SDs. Statistical analysis was performed using one-way analysis of variance with Dunn's multiple comparison test $\left({ }^{*} p<0.05,{ }^{* *} p<0.01\right.$, ${ }^{* * *} p<0.001$, and $\left.{ }^{* * * *} p<0.0001\right)$.

hydroxide), AP205-OPS, or Q $\beta$-OPS on days 0,14 , and 28 (Fig. 5(a)). Antibody titers against S. flexneri LPS were measured by ELISA. After a single priming dose, no significant differences were found among groups on day 14 postimmunization. However, after one and two additional boosting doses, AP205-OPS or Q $\beta$-OPS-vaccinated mice had significantly higher antibody responses. Low levels of LPS-specific immunoglobulin (IgG) were detected in sera from mice vaccinated with SC-OPS with or without Alum (Fig. 5(b)). LPS-specific antibody titers elicited by the nanovaccines peaked 2 weeks after the third immunization and remained high 4 weeks thereafter (Fig. 5(c)). Also, the robust higher titers of four IgG subtypes against $S$. flexneri 2 a strain 301 LPS in AP205-OPS and Q $\beta$-OPS groups indicated that both humoral and cellular immune responses could be induced by the nanovaccines (Fig. S7 in the ESM). To assess whether unconjugated VLP affected the immune response against the polysaccharide antigen, SC-VLP and SC-OPS were mixed and used to immunize the mice (Fig. S8 in the ESM). The antibody response elicited by the SC-OPS + SC-VLP mixture was significantly weaker than that elicited by the AP205-OPS or $\mathrm{Q} \beta$-OPS nanovaccines, indicating that the physical mixing of VLP with SC-OPS could not enhance the immune response against this antigen (Fig. S8 in the ESM). Notably, antibody titers against OPS and SC-OPS did not increase significantly following immunization with Alum adjuvant (Fig. S9 in the ESM).

We next evaluated the bactericidal activity of sera from 
(a)

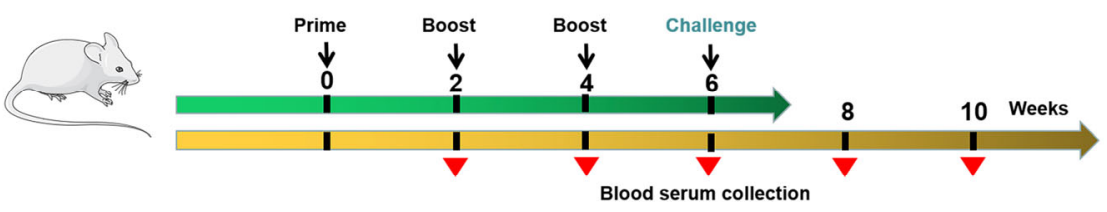

(b)

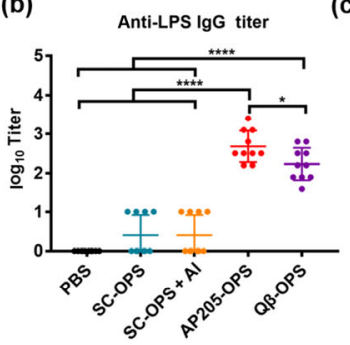

(e)

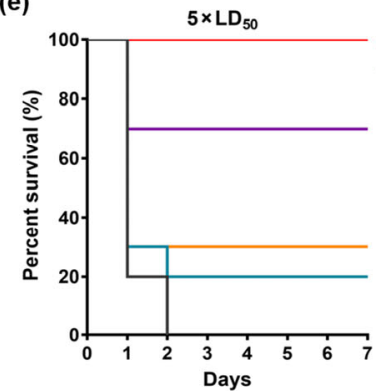

(c)

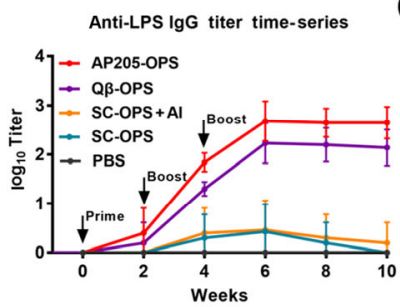

(d)
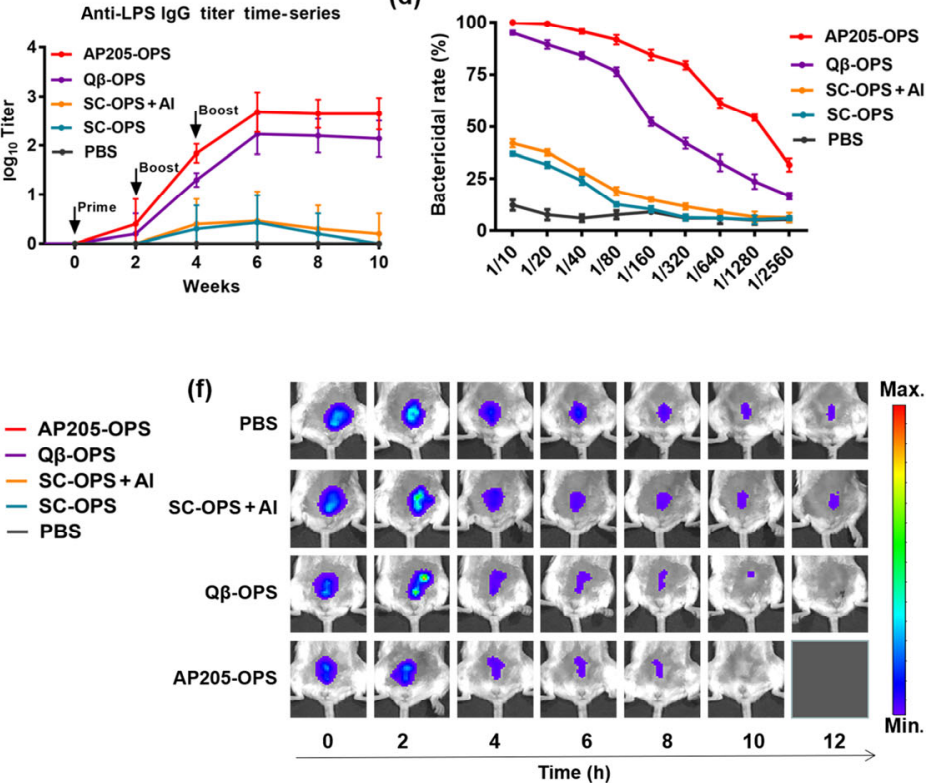

Figure 5 Nanovaccines elicited enhanced humoral immunity and afforded robust protection against S. flexneri infection. (a) Schematic illustration of the immunization study. (b) Total serum IgG against S. flexneri 2a strain 301 LPS determined on day 42. Data are presented as means \pm SDs $(n=10)$. Statistical analysis was performed using the Kruskal-Wallis test $\left({ }^{*} p<0.05,{ }^{* * * *} p<0.0001\right)$. (c) LPS-specific IgG titers from individual mice were analyzed over time by ELISA $(n=10)$. (d) Serum bactericidal activity was determined on day $42(n=10)$. (e) Survival of different groups of mice challenged with $4.35 \times 10^{7}$ $\mathrm{CFU}$ per mouse $\left(5 \times \mathrm{LD}_{50}\right)$ of $S$. flexneri 2 a strain 301 cells on day 42 . Survival was monitored over the subsequent 7 days $(n=10)$. (f) On day 42 , mice were injected intraperitoneally with luciferase-labeled S. flexneri 2a strain 301 (the pXen-18 plasmid was introduced into the strain 301). Bactericidal activity was observed via luciferase activity measured at different time points $(n=3)$.

vaccinated mice using complement sterilization experiments. As shown in Fig. 5(d), sera from vaccinated mice immunized with AP205-OPS and Q $\beta$-OPS showed a high level of bactericidal activity against $S$. flexneri 2 a 301 strain compared to the serum from an SC-OPS-vaccinated mouse. To confirm the protective efficacy of the nanovaccines, 10 mice from each vaccination group were inoculated intraperitoneally with approximately $4.35 \times 10^{7}$ colony-forming unit (CFU)/mouse $\left(5 \times\right.$ median lethal dose $\left.\left(\mathrm{LD}_{50}\right)\right)$ of $S$. flexneri 2 a strain 301 cells 6 weeks post-immunization. Following this high-dose challenge, the 7-day survival rates of the mice immunized with AP205OPS and Q $\beta$-OPS were $100 \%$ and $70 \%$, respectively. Most of the mice immunized with other formulations mice died within 2 days and the 7 -day survival rates did not exceed $40 \%$ (Fig. 5(e)). To observe the bactericidal activity of antibodies elicited by the nanovaccines visually, we intradermally injected luciferase-labeled S. flexneri 2a strain 301 into the abdomens of immunized mice and measured luciferase activity at different time points. We observed that bacterial clearance in AP205OPS- and Q $\beta$-OPS-immunized mice was significantly faster than in other mice (Fig. 5(f)). These results demonstrated that AP205-OPS and Q $\beta$-OPS were significantly more effective than SC-OPS and OPS (with or without Alum adjuvant) in inducing humoral immune responses against bacterial polysaccharide antigens that afforded sufficient protection against challenge with $S$. flexneri.

\section{Conclusion}

In summary, we have established a modular method for preparing two kinds of uniform and stabile nanoconjugate vaccines using the SpyTag/SpyCatcher system and PGCT. This method enables the uncoupled expression of scaffold and antigen in E. coli and the host bacterium, respectively, ensuring structural integrity of both scaffold and polysaccharides antigen. The construction and production of antigen and scaffold could be optimized using different expression systems. It should be emphasized that the polysaccharide antigens in our nanovaccines were synthesized by bacteria themselves (without artificial intervention), guaranteeing their natural conformation.

In addition, compared with vaccines derived from traditional PGCT, the nanoconjugate vaccine effectively increased the numbers of Tfh and GC B cells in dLNs, triggering an efficient adaptive immune response, and elicited strong prophylactic effects against infection. Using this method, we can produce validated scaffolds in advance and the corresponding antigens as needed, significantly shortening vaccine preparation times. It also bears to mention that this method may find applications for various pathogenic bacterial, especially multiple drug-resistant bacteria such as Enterococcus faecium, Staphylococcus aureus, Klebsiella pneumoniae, Acinetobacter baumannii, Pseudomonas aeruginosa, and Enterobacter (ESKAPE) pathogens.

\section{Experimental}

\subsection{Cloning, expression, and purification of SpyTag- VLPs}

Genes encoding SpyTag-VLPs were synthesized by GenScript (Nanjing, China) and cloned into the pET28a vector in the following order: SpyTag, GSGTAGGGSGS linker, AP205 coat 
protein 3 or $\mathrm{Q} \beta$, and C-terminal His 6 tag. E. coli BL21 cells were transformed with the resulting construct and cells were cultured on Luria-Bertani (LB) agar plates containing kanamycin $(50 \mu \mathrm{g} / \mathrm{mL})$ at $37^{\circ} \mathrm{C}$ for $12 \mathrm{~h}$. A single colony was picked and used to inoculate $\mathrm{LB}$ medium $(10 \mathrm{~mL})$ containing kanamycin $(50 \mu \mathrm{g} / \mathrm{mL})$. The culture was incubated at $37^{\circ} \mathrm{C}$ with $200 \mathrm{rpm}$ shaking for $16 \mathrm{~h}$. The culture was then diluted 1:100 in LB medium ( $1 \mathrm{~L}$ ) containing $50 \mu \mathrm{g} / \mathrm{mL}$ of kanamycin and incubated with $200 \mathrm{rpm}$ shaking at $37{ }^{\circ} \mathrm{C}$. When the cultures reached an $A_{600 \mathrm{~nm}}$ of $0.6-0.8$, they were induced with IPTG and grown at $200 \mathrm{rpm}$ shaking and $30^{\circ} \mathrm{C}$ for $10-12 \mathrm{~h}$. Cultures were harvested by centrifugation at $8,000 \mathrm{~g}$ for $10 \mathrm{~min}$ and pellets were resuspended in buffer $\mathrm{Al}(20 \mathrm{mM}$ Tris. $\mathrm{HCl}$, $\mathrm{pH} 7.8$, containing $150 \mathrm{mM} \mathrm{NaCl}, 70 \mathrm{mM}$ imidazole, and $0.1 \%(\mathrm{v} / \mathrm{v})$ Tween-20). Cells were homogenized and centrifuged twice $(10,000 \mathrm{~g})$ at $4{ }^{\circ} \mathrm{C}$ for $10 \mathrm{~min}$. The supernatant was incubated with $250 \mathrm{U}$ of benzonase at $25{ }^{\circ} \mathrm{C}$ for $5 \mathrm{~min}$. A His-Tag purification resin column (Roche, Basel, Switzerland) was pre-equilibrated with buffer A1. Bound protein was eluted in $100 \%$ buffer B1 containing $50 \mathrm{mM}$ Tris. $\mathrm{HCl}$ ( $\mathrm{pH} 7.8$ ), $2 \mathrm{M}$ imidazole, $150 \mathrm{mM} \mathrm{NaCl}$, and $0.1 \%$ Tween-20. Before elution, the column was washed with $50 \mathrm{mM}$ Tris. $\mathrm{HCl}(\mathrm{pH} 7.8)$ containing $150 \mathrm{mM} \mathrm{NaCl}, 100 \mathrm{mM}$ imidazole, and $0.1 \%$ Tween-20. The eluate was centrifuged at $17,000 \mathrm{~g}$ and $4{ }^{\circ} \mathrm{C}$ for $30 \mathrm{~min}$. It was transferred to a $100 \mathrm{kDa}$ MWCO membrane and dialyzed overnight at $4{ }^{\circ} \mathrm{C}$ against a 1,000 -fold volumetric excess of $25 \mathrm{mM}$ sodium citrate $(\mathrm{pH} 8.0)$ containing $50 \mathrm{mM}$ glycine and $0.1 \%$ Tween-20. Dialysis was repeated for an additional $3 \mathrm{~h}$. Finally, the sample was dialyzed in PBS for $5 \mathrm{~h}$. After dialysis, the sample was centrifuged once more at 17,000 g, and $4{ }^{\circ} \mathrm{C}$ for $10 \mathrm{~min}$ to remove any aggregates.

\subsection{Cloning, expression and purification of SC-OPS}

A gene encoding SpyCatcher was synthesized by GenScript. The pET-PgIL-SpyCatcher ${ }^{473 \mathrm{C}}$ vector was derived by polymerase chain reaction (PCR) from pET-pglL-CTB ${ }^{4573}$. The pET-PgILSpyCatcher ${ }^{4573 C}$ vector is organized thus: PglL, Tac promoter, SpyCatcher, GGSG, glycosylation sequence 4573, His 6 tag. S. flexneri strain 301DWP cells were transformed with pETPgIL-SpyCatcher ${ }^{4573}$ and plated on LB agar plates containing kanamycin $(50 \mu \mathrm{g} / \mathrm{mL})$. The plates were incubated at $37^{\circ} \mathrm{C}$ for $12 \mathrm{~h}$. A single colony was picked into LB medium $(5 \mathrm{~mL})$ containing $50 \mu \mathrm{g} / \mathrm{mL}$ kanamycin and incubated at $37{ }^{\circ} \mathrm{C}$ with $200 \mathrm{rpm}$ shaking for $16 \mathrm{~h}$. For protein expression, cells were cultured in LB containing kanamycin $(50 \mu \mathrm{g} / \mathrm{mL})$ at $37^{\circ} \mathrm{C}$ until an $A_{600 \mathrm{~nm}}$ of approximately 0.6 was reached. Then, $1 \mathrm{mM}$ IPTG was added to induce protein expression at $30^{\circ} \mathrm{C}$ with $200 \mathrm{rpm}$ shaking for $10-12 \mathrm{~h}$. The culture pellet was resuspended in buffer $\mathrm{A} 1(20 \mathrm{mM}$ Tris. $\mathrm{HCl}, \mathrm{pH} 7.5$, containing $0.5 \mathrm{M} \mathrm{NaCl}$ and $10 \mathrm{mM}$ imidazole). Cells were homogenized and centrifuged twice $(10,000 \mathrm{~g})$ at $4{ }^{\circ} \mathrm{C}$ for $10 \mathrm{~min}$. The supernatant was applied to a His-tag purification resin column and washed with five column volumes of buffer A1. Bound protein was eluted with $100 \%$ buffer B1 (20 mM Tris- $\mathrm{HCl}, \mathrm{pH}$ 7.5 , containing $0.5 \mathrm{M} \mathrm{NaCl}$ and $0.5 \mathrm{M}$ imidazole). The eluate was concentrated by ultrafiltration (Millipore Amicon ${ }^{\circledR}$ Ultra-0.5) to approximately $5 \mathrm{~mL}$ and further purified through a Superdex-200 column (GE Healthcare, Piscataway, NJ, USA) in a mobile phase consisting of PBS. Fractions were collected and analyzed by $12 \%$ SDS-PAGE.

\subsection{Generation and purification of nanoconjugate} vaccines

The purified SC-OPS was conjugated to SpyTag-AP205 or SpyTag-Q $\beta$ in vitro to prepare the nanoconjugate vaccines.
Different molar ratios of SpyTag-VLPs/SC-OPS were tested. For vaccine generation, SpyTag-VLPs were mixed with SC-OPS at a molar ratio of $1: 1.5$ and reacted overnight at $4{ }^{\circ} \mathrm{C}$. The conjugated SpyTag-VLPs + SC-OPS was then purified using a Superdex-200 column (GE Healthcare). SpyTag-VLPs + SC-OPS eluted in the void volume.

\subsection{Western blotting}

Western blotting was performed as described previously [40]. All proteins were boiled under reducing conditions in $2 \times$ SDS buffer and then separated using 12\% SDS-PAGE gels. We used a horseradish peroxidase (HRP)-conjugated anti-His ${ }_{6}$ antibody (Abmart, Berkeley Heights, NJ, USA) to detect His ${ }_{6}$-containing proteins and an S. flexneri 2a OPS-specific antibody (Denka Seiken, Tokyo, Japan) to detect glycoproteins. HRP-labeled anti-rabbit IgG (Transgen Biotech, Beijing, China) was used as the secondary antibody for the OPS-specific antibody.

\subsection{Transmission electron microscopy}

The samples were applied to freshly glow-discharged 200-mesh copper grids for $1 \mathrm{~min}$. Filter paper was used to absorb excess liquid. The grids were stained with $2 \%(\mathrm{w} / \mathrm{v})$ uranyl acetate for $45 \mathrm{~s}$, and then air-dried. Negatively stained grids were imaged on a Hitachi HT7700 microscope operating at $80 \mathrm{kV}$.

\subsection{Dynamic light scattering}

DLS was used to characterize the hydrodynamic diameters of nanoconjugate vaccines and ST-VLPs using a Zetasizer Pro instrument (Malvern Panalytical, Malvern, UK). Briefly, protein samples were diluted to $200 \mu \mathrm{g} / \mathrm{mL}$ in PBS and centrifuged for $10 \mathrm{~min}$ at $16,000 \mathrm{~g}$ and $4{ }^{\circ} \mathrm{C}$ to remove any aggregates. Each sample $(1 \mathrm{~mL})$ was placed into a disposable cuvette and installed in the DLS chamber. Each sample was measured in triplicate at $20^{\circ} \mathrm{C}$ and intensity distributions were plotted.

\subsection{Activation of BMDCs by nanovaccines in vitro}

BMDCs were separated from bone marrow cells isolated from the tibias and femurs of BALB/c mice. The cells were cultured for 6 days in RPMI 1640 medium containing $10 \%$ fetal bovine serum, $1 \%$ penicillin-streptomycin, granulocyte-macrophage colony-stimulating factor $(10 \mathrm{ng} / \mathrm{mL})$, and IL-4 $(20 \mathrm{ng} / \mathrm{mL})$. The culture medium was replaced every other day. After 5 days, the cells were harvested as immature DCs for further evaluation.

BMDCs were plated at $1.0 \times 10^{5}$ cells/well in a 24 -well plate, and then stimulated with OPS, SC-OPS, AP205-OPS, and $\mathrm{Q} \beta$-OPS for $24 \mathrm{~h}$. Non-stimulated BMDCs were used as a negative control. Cells were collected and stained with the following antibodies at $4{ }^{\circ} \mathrm{C}$ for $30 \mathrm{~min}$ (all from eBioscience, San Diego, CA, USA): fluorescein isothiocyanate (FITC)conjugated anti-mouse $\mathrm{CD} 11 \mathrm{c}$, phycoerythrin (PE)-conjugated anti-mouse CD40, allophycocyanin (APC)-conjugated anti-mouse CD80, APC-conjugated anti-mouse CD86, and Alexa Fluor (AF) 700-conjugated anti-mouse MHC II. After washing with staining buffer (eBioscience), the cells were resuspended in $300 \mu \mathrm{L}$ of staining buffer and analyzed using a CytoFLEX LX flow cytometer (Beckman Coulter, Brea, CA, USA). The cell culture supernatants of BMDCs were collected to assess the levels of IL-6, TNF- $\alpha$, and IL-1 $\beta$ using ELISA kits according to the protocol given by the manufacturer (Dakewe, Shenzhen, China).

\subsection{Lymph node imaging assay}

SC-OPS, AP205-OPS, and Q $\beta$-OPS were labeled with Cy7-SE (Fanbo Biochemicals, Beijing, China) at $4{ }^{\circ} \mathrm{C}$ overnight. Unbound 
dye was removed using a $10-\mathrm{kDa}$ cutoff centrifugal filter (Merck, Kenilworth, NJ, USA) four times. The fluorescence intensity of each sample was tested in vitro to ensure that the initial fluorescence intensity of each group was similar. BALB/c mice were subcutaneously injected in the tail base with Cy7-labeled sample $(100 \mu \mathrm{L})$ and then imaged using an IVIS spectrum in vivo imaging system (PerkinElmer, Waltham, MA, USA) at different time points.

\subsection{Flow cytometry}

All antibodies for flow cytometry were from eBiocsience unless indicated otherwise. Prior to flow cytometry, dLNs were harvested to prepare single-cell suspensions. The single-cell suspension was incubated with APC-conjugated anti-mouse $\mathrm{CD} 3$ and FITC-conjugated anti-mouse CD4 at $4{ }^{\circ} \mathrm{C}$ for $30 \mathrm{~min}$. For analysis of the proportions of Tfh cells and GC B cells, the single-cell suspension from dLNs was stained with different combinations of flow cytometry antibodies including APCconjugated anti-mouse B220, AF488-conjugated anti-mouse GL-7, PE-conjugated anti-mouse CD95, FITC-conjugated anti-mouse $\mathrm{CD} 4$, PE-conjugated anti-mouse PD-1, and APCconjugated anti-mouse CXCR5 (Biolegend, San Diego, CA, USA) at $4{ }^{\circ} \mathrm{C}$ for $30 \mathrm{~min}$. After washing with staining buffer, the cells were resuspended in $300 \mu \mathrm{L}$ of staining buffer and analyzed using a CytoFLEX LX flow cytometer.

\subsection{Lymph node immunofluorescence microscopy}

The lymph nodes of the mice were removed 7 days after immunization and fixed with $4 \%$ paraformaldehyde. The samples were washed with PBS and embedded. The lymph nodes were sliced into $100 \mu \mathrm{m}$ thick sections. The sections were blocked with $5 \%$ bovine serum albumin for $30 \mathrm{~min}$, and then stained with anti-B220 and anti-Ki67 (Servicebio, Wuhan, China) at $4{ }^{\circ} \mathrm{C}$ overnight. The sections were washed with PBS and then stained with 4',6-diamidino-2-phenylindole (DAPI) (Servicebio, Wuhan, China) at room temperature for $30 \mathrm{~min}$. The sections were washed with PBS and mounted on a glass slide.

\subsection{Mouse vaccination}

Specific-pathogen-free female mice were purchased from Beijing Vital River Laboratory Animal Technology Co., Ltd. (Beijing, China) and were housed in the Animal Center of the Academy of Military Medical Sciences. All animal experiments were approved and conducted in accordance with the institutional guidelines of the Academy of Military Medical Sciences and with approval from the Institutional Animal Care and Use Committee (approval ID IACUC-DWZX-2019-401). $\mathrm{BALB} / \mathrm{c}$ mice (6-8 weeks old) were randomly divided into five groups and immunized three times at 2-week intervals by subcutaneous injection with $100 \mu \mathrm{L}$ of PBS, SC-OPS, SC-OPS + Alum $\left(100 \mu \mathrm{g} / \mathrm{mouse} \mathrm{Al}(\mathrm{OH})_{3}\right.$, Invivogen, San Diego, CA, USA), AP205-OPS, or Q $\beta$-OPS. The dose of polysaccharide was $2.5 \mu \mathrm{g}$ per injection. Blood samples were taken by tail snip every 2 weeks and serum was stored at $4{ }^{\circ} \mathrm{C}$.

\subsection{ELISA}

LPS $(10 \mu \mathrm{g} /$ well) was coated in 96-well plates in carbonate buffer $\left(50 \mathrm{mM} \mathrm{Na} \mathrm{CO}_{3}-\mathrm{NaHCO}_{3}, \mathrm{pH} 9.6\right)$ at $4{ }^{\circ} \mathrm{C}$ overnight. After washing the plates three times with wash buffer (PBS containing $0.05 \%$ Tween-20), plates were blocked with $5 \%$ $(\mathrm{w} / \mathrm{v})$ skim milk in wash buffer at $37^{\circ} \mathrm{C}$ for $2 \mathrm{~h}$. Plates were washed three times with wash buffer and incubated (in duplicate) with two-fold serially diluted serum samples in dilution buffer (wash buffer containing $0.5 \%$ skim milk powder) at $37^{\circ} \mathrm{C}$ for $1 \mathrm{~h}$. After washing the plates, HRP-conjugated goat anti-mouse IgG $(100 \mu \mathrm{L}$, Abcam, Cambridge, UK; 1:15,000 dilution in dilution buffer) was added to the plates. The plates were incubated at $37^{\circ} \mathrm{C}$ for another $1 \mathrm{~h}$. After washing with wash buffer, tetramethylbenzidine solution $(100 \mu \mathrm{L}$, CWBio, Beijing, China) was added to each well. The reaction was stopped with stop solution. Absorbance was measured at $450 \mathrm{~nm}$ using a microplate spectrophotometer.

\subsection{Serum bactericidal assay}

S. flexneri 2a strain 301 cells were cultured at $37^{\circ} \mathrm{C}$ until an $A_{600 \mathrm{~nm}}$ of 2.0 was reached, and then diluted with normal saline to $2.0 \times 10^{4} \mathrm{CFU} / \mathrm{mL}$. Immune sera from each group of mice were mixed. Complement components were inactivated by heat treatment at $56{ }^{\circ} \mathrm{C}$ for $30 \mathrm{~min}$. The inactivated immune sera were diluted to varying concentrations and an aliquot $(10 \mu \mathrm{L})$ of each dilution was added to a tube. Then, diluted S. flexneri 2 a cells $(10 \mu \mathrm{L})$ were added to each dilution and incubated at room temperature for $1 \mathrm{~h}$. Thereafter, complement (20 $\mu \mathrm{L}$, Pel-Freez, Brown Deer, WI, USA) was added and incubated at $37^{\circ} \mathrm{C}$ for $1 \mathrm{~h}$. Finally, the mixtures were plated on $\mathrm{LB}$ agar and incubated at $37^{\circ} \mathrm{C}$ overnight. The CFUs on each plate were calculated to assess bactericidal activity.

\subsection{Mouse model of $S$. flexneri 2a infection}

The inoculum was prepared as previously mentioned. Each mouse was intraperitoneally injected with $4.35 \times 10^{7} \mathrm{CFU}$ of $S$. flexneri 2 a strain 301,42 days post-immunization $(n=10$ per group), and their survival was monitored continuously for 7 days.

\subsection{Statistical analysis}

All analyses were performed using GraphPad Prism 7.0 statistical software (GraphPad Inc, San Diego, CA, USA). Data were analyzed using the Kruskal-Wallis test or one-way ANOVA with Dunn's multiple comparison test. Results were expressed as means \pm SDs. Values of $p<0.05$ were considered statistically significant $\left({ }^{* * *} p<0.0001,{ }^{* * *} p<0.001,{ }^{* *} p<0.01\right.$, and ${ }^{*} p<$ $0.05)$.

\section{Acknowledgements}

This work was supported by the National Natural Science Foundation of China (Nos. 81930122 and U20A20361) and the National Key Research and Development Project of China (No. 2021YFC2102101).

Electronic Supplementary Material: Supplementary material (Figs. S1-S9) is available in the online version of this article at https://doi.org/10.1007/s12274-021-3713-4.

\section{References}

[1] Irvine, D. J.; Swartz, M. A.; Szeto, G. L. Engineering synthetic vaccines using cues from natural immunity. Nat. Mater. 2013, 12, 978-990.

[2] Karch, C. P.; Burkhard, P. Vaccine technologies: From whole organisms to rationally designed protein assemblies. Biochem. Pharmacol. 2016, 120, 1-14.

[3] Schmidt, S. T.; Foged, C.; Korsholm, K. S.; Rades, T. Liposome-based adjuvants for subunit vaccines: Formulation strategies for subunit antigens and immunostimulators. Pharmaceutics 2016, 8, 7.

[4] Poteet, E.; Lewis, P.; Chen, C. Y.; Ho, S. O.; Do, T.; Chiang, S.; Labranche, C.; Montefiori, D.; Fujii, G.; Yao, Q. Z. Toll-like receptor 3 adjuvant in combination with virus-like particles elicit a humoral response against HIV. Vaccine 2016, 34, 5886-5894. 
[5] Vartak, A.; Sucheck, S. J. Recent advances in subunit vaccine carriers Vaccines 2016, 4, 12.

[6] Dowling, D. J.; Scott, E. A.; Scheid, A.; Bergelson, I.; Joshi, S.; Pietrasanta, C.; Brightman, S.; Sanchez-Schmitz, G.; van Haren, S. D.; Ninkovic, J. et al. Toll-like receptor 8 agonist nanoparticles mimic immunomodulating effects of the live BCG vaccine and enhance neonatal innate and adaptive immune responses. J. Allergy Clin. Imтипоl. 2017, 140, 1339-1350.

[7] Lynn, G. M.; Laga, R.; Darrah, P. A.; Ishizuka, A. S.; Balaci, A. J.; Dulcey, A. E.; Pechar, M.; Pola, R.; Gerner, M. Y.; Yamamoto, A. et al. In vivo characterization of the physicochemical properties of polymer-linked TLR agonists that enhance vaccine immunogenicity. Nat. Biotechnol. 2015, 33, 1201-1210.

[8] Ebrahimian, M.; Hashemi, M.; Maleki, M.; Hashemitabar, G.; Abnous, K.; Ramezani, M.; Haghparast, A. Co-delivery of dual toll-like receptor agonists and antigen in poly(lactic-Co-glycolic) acid/polyethylenimine cationic hybrid nanoparticles promote efficient in vivo immune responses. Front. Immunol. 2017, 8, 1077.

[9] Christensen, D. Vaccine adjuvants: Why and how. Hum. Vaccin. Immunother 2016, 12, 2709-2711.

[10] Banday, A. H.; Jeelani, S.; Hruby, V. J. Cancer vaccine adjuvantsrecent clinical progress and future perspectives. Immunopharmacol. Immunotoxicol. 2015, 37, 1-11.

[11] Xu, H.; Huang, J.; Liu, Z. L.; Li, X.; Wang, K. F.; Feng, E. L.; Wu, J.; Zhu, L.; Yao, K. H.; Pan, C. et al. Expression of Bordetella pertussis antigens fused to different vectors and their effectiveness as vaccines. Vaccines (Basel) 2021, 9, 542.

[12] Reddy, S. T.; van der Vlies, A. J.; Simeoni, E.; Angeli, V.; Randolph, G. J.; O'Neil, C. P.; Lee, L. K.; Swartz, M. A.; Hubbell, J. A. Exploiting lymphatic transport and complement activation in nanoparticle vaccines. Nat. Biotechnol. 2007, 25, 1159-1164.

[13] Irvine, D. J.; Hanson, M. C.; Rakhra, K.; Tokatlian, T. Synthetic nanoparticles for vaccines and immunotherapy. Chem. Rev. 2015, $115,11109-11146$.

[14] Molino, N. M.; Neek, M.; Tucker, J. A.; Nelson, E. L.; Wang, S. W. Display of DNA on nanoparticles for targeting antigen presenting cells. ACS Biomater. Sci. Eng. 2017, 3, 496-501.

[15] Bachmann, M. F.; Jennings, G. T. Vaccine delivery: A matter of size, geometry, kinetics and molecular patterns. Nat. Rev. Immunol. 2010, 10, 787-796.

[16] Win, S. J.; Ward, V. K.; Dunbar, P. R.; Young, S. L.; Baird, M. A. Cross-presentation of epitopes on virus-like particles via the MHC I receptor recycling pathway. Immunol. Cell Biol. 2011, 89, 681-688.

[17] Lin, A. Y.; Lunsford, J.; Bear, A. S.; Young, J. K.; Eckels, P.; Luo, L.; Foster, A. E.; Drezek, R. A. High-density sub-100-nm peptide-gold nanoparticle complexes improve vaccine presentation by dendritic cells in vitro. Nanoscale Res. Lett. 2013, 8, 72.

[18] An, M.; Li, M.; Xi, J.C.; Liu, H. P. Silica nanoparticle as a lymph node targeting platform for vaccine delivery. ACS Appl. Mater. Interfaces 2017, 9, 23466-23475.

[19] Kang, S.; Ahn, S.; Lee, J.; Kim, J. Y.; Choi, M.; Gujrati, V.; Kim, H.; Kim, J.; Shin, E. C.; Jon, S. Effects of gold nanoparticle-based vaccine size on lymph node delivery and cytotoxic T-lymphocyte responses. J. Control. Release 2017, 256, 56-67.

[20] Rahimian, S.; Kleinovink, J. W.; Fransen, M. F.; Mezzanotte, L.; Gold, H.; Wisse, P.; Overkleeft, H.; Amidi, M.; Jiskoot, W.; Lowik, C. W. et al. Near-infrared labeled, ovalbumin loaded polymeric nanoparticles based on a hydrophilic polyester as model vaccine: In vivo tracking and evaluation of antigen-specific $\mathrm{CD} 8^{+} \mathrm{T}$ cell immune response. Biomaterials 2015, 37, 469-477.

[21] Wen, R.; Umeano, A. C.; Chen, P. P.; Farooqi, A. A. Polymer-based drug delivery systems for cancer. Crit. Rev. Ther. Drug Carrier Syst. 2018, 35, 521-553.

[22] De Gregorio, E.; Rappuoli, R. From empiricism to rational design: A personal perspective of the evolution of vaccine development. Nat. Rev. Immunol. 2014, 14, 505-514.

[23] Pushko, P.; Pumpens, P.; Grens, E. Development of virus-like particle technology from small highly symmetric to large complex virus-like particle structures. Intervirology 2013, 56, 141-165.

[24] Smith, M. T.; Hawes, A. K.; Bundy, B. C. Reengineering viruses and virus-like particles through chemical functionalization strategies. Curr. Opin. Biotechnol. 2013, 24, 620-626.

[25] Mateu, M. G. Virus engineering: Functionalization and stabilization. Protein Eng. Des. Sel. 2011, 24, 53-63.

[26] Strable, E.; Finn, M. G. Chemical modification of viruses and viruslike particles. In Viruses and Nanotechnology. Manchester, M.; Steinmetz, M. F., Eds.; Springer: Berlin, 2009; pp 1-21.

[27] Sletten, E. M.; Bertozzi, C. R. Bioorthogonal chemistry: Fishing for selectivity in a sea of functionality. Angew. Chem., Int. Ed. 2009, 48, 6974-6998.

[28] Zakeri, B.; Fierer, J. O.; Celik, E.; Chittock, E. C.; Schwarz-Linek, U.; Moy, V. T.; Howarth, M. Peptide tag forming a rapid covalent bond to a protein, through engineering a bacterial adhesin. Proc. Natl. Acad. Sci. USA 2012, 109, E690-E697.

[29] Li, L.; Fierer, J. O.; Rapoport, T. A.; Howarth, M. Structural analysis and optimization of the covalent association between SpyCatcher and a peptide Tag. J. Mol. Biol. 2014, 426, 309-317.

[30] Brune, K. D.; Leneghan, D. B.; Brian, I. J.; Ishizuka, A. S.; Bachmann, M. F.; Draper, S. J.; Biswas, S.; Howarth, M. Plug-and-display: Decoration of virus-like particles via isopeptide bonds for modular immunization. Sci. Rep. 2016, 6, 19234.

[31] Janitzek, C. M.; Matondo, S.; Thrane, S.; Nielsen, M. A.; Kavishe, R.; Mwakalinga, S. B.; Theander, T. G.; Salanti, A.; Sander, A. F. Bacterial superglue generates a full-length circumsporozoite protein virus-like particle vaccine capable of inducing high and durable antibody responses. Malar. J. 2016, 15, 545.

[32] Brune, K. D.; Buldun, C. M.; Li, Y. Y.; Taylor, I. J.; Brod, F.; Biswas, S.; Howarth, M. Dual plug-and-display synthetic assembly using orthogonal reactive proteins for twin antigen immunization. Bioconjugate Chem. 2017, 28, 1544-1551.

[33] Thrane, S.; Janitzek, C. M.; Matondo, S.; Resende, M.; Gustavsson, T.; de Jongh, W. A.; Clemmensen, S.; Roeffen, W.; van de VegteBolmer, M.; van Gemert, G. J. et al. Bacterial superglue enables easy development of efficient virus-like particle based vaccines. $J$. Nanobiotechnol. 2016, 14, 30 .

[34] Sáfadi, M. A.; Bettinger, J. A.; Maturana, G. M.; Enwere, G.; Borrow, R.; Global Meningococcal, I. Evolving meningococcal immunization strategies. Exp. Rev. Vaccin. 2015, 14, 505-517.

[35] Martin, L. B.; Simon, R.; MacLennan, C. A.; Tennant, S. M.; Sahastrabuddhe, S.; Khan, M. I. Status of paratyphoid fever vaccine research and development. Vaccine 2016, 34, 2900-2902.

[36] Bröker, M.; Berti, F.; Costantino, P. Factors contributing to the immunogenicity of meningococcal conjugate vaccines. Hum. Vacc. Immunother. 2016, 12, 1808-1824.

[37] Kuberan, B.; Linhardt, R. J. Carbohydrate based vaccines. Curr. Org. Chem. 2000, 4, 635-677.

[38] Pan, C.; Wu, J.; Qing, S.; Zhang, X.; Zhang, L. L.; Yue, H.; Zeng, M.; Wang, B.; Yuan, Z.; Qiu, Y. F. et al. Biosynthesis of self-assembled proteinaceous nanoparticles for vaccination. Adv. Mater. 2020, 32, 2002940.

[39] Peng, Z. H.; Wu, J.; Wang, K. F.; Li, X.; Sun, P.; Zhang, L. L.; Huang, J.; Liu, Y.; Hua, X. T.; Yu, Y. S. et al. Production of a promising biosynthetic self-assembled nanoconjugate vaccine against Klebsiella pneumoniae serotype $\mathrm{O}_{2}$ in a general Escherichia coli host. Adv. Sci., in press, DOI: 10.1002/advs.202100549.

[40] Pan, C.; Sun, P.; Liu, B.; Liang, H. Y.; Peng, Z. H.; Dong, Y.; Wang, D. S.; Liu, X. K.; Wang, B.; Zeng, M. et al. Biosynthesis of conjugate vaccines using an O-linked glycosylation system. mBio 2016, 7, e00443-16.

[41] Hanson, C. M.; George, A. M.; Sawadogo, A.; Schreiber, B. Is freezing in the vaccine cold chain an ongoing issue? A literature review. Vaccine 2017, 35, 2127-2133.

[42] Paulis, L. E.; Mandal, S.; Kreutz, M.; Figdor, C. G. Dendritic cell-based nanovaccines for cancer immunotherapy. Curr. Opin. Immunol. 2013, 25, 389-395.

[43] Vinuesa, C. G.; Linterman, M. A.; Yu, D.; MacLennan, I. C. M. Follicular helper T cells. Annu. Rev. Immunol. 2016, 34, 335-368.

[44] McHeyzer-Williams, L. J.; Milpied, P. J.; Okitsu, S. L.; McHeyzerWilliams, M. G. Class-switched memory B cells remodel BCRs within secondary germinal centers. Nat. Immunol. 2015, 16, 296-305. 